\title{
LiDAR resolution for catchment-inclusive hydrodynamic models
}

\author{
Graeme $\mathrm{Smart}^{1}$ \\ ${ }^{1}$ National Institute of Water \& Atmospheric Research (NIWA), Christchurch, New Zealand
}

\begin{abstract}
Hydrodynamic models are usually based on LiDAR but there is little information on the LiDAR resolution required for appropriate model accuracy. In this study, algorithms to prepare DEM and roughness grids suitable for hydrodynamic modelling of both catchment and floodplain are applied to low, medium and high point-density LiDAR. The medium resolution LiDAR (9 points $/ \mathrm{m}^{2}$ ) provided elevation and roughness grids sufficiently accurate for hydrodynamic flood mapping of urban and rural floodplains. Low-resolution LiDAR (3 points $/ \mathrm{m}^{2}$ ) is considered adequate for hill catchments. Attention is required where narrow-crested control structures exist. Mapping and upscaling are discussed.
\end{abstract}

\section{Introduction}

LiDAR is used in flood modelling for determining topography, ground cover, surface roughness and channel networks and for revealing hydraulic controls such as bridges, structure crest levels and levees. Scan densities from LiDAR sorties for hydraulic modelling typically range from 1-20 points $/ \mathrm{m}^{2}$. LiDAR "hits" may be from the ground, buildings or vegetation. For numerical hydraulic modelling purposes, LiDAR hits are converted to digital elevation models (DEMs) of the topography and may be used for roughness mapping [1]. While DEMs have been resampled at different resolutions to evaluate the effects of different grid cell sizes on flood simulations $[2,3]$ there is little information on the minimum resolution of raw LiDAR to supply sufficient information for accurate flood mapping at a given cell size. Rain-on-grid hydrodynamic models now allow model domains to be extended into catchment areas [1] but the high LiDAR scan densities required for urban areas with infrastructure are unlikely to be necessary for e.g. hill country catchments. This paper investigates the effects of LiDAR resolution in relation to hydrodynamic model DEMs and roughness maps.

\section{Methodology}

Appropriate LiDAR resolution is investigated with case studies in an area that is representative of a wide range of New Zealand conditions. Three LiDAR resolutions with nominal point densities of 1,4 and 8 points per square metre are investigated. The point cloud LiDAR data at the different resolutions is used to extract representative hydraulic modelling DEMs and representative hydraulic modelling roughness maps. The DEMs are studied to investigate narrow channel continuity, hydraulic controls and structure crest 
levels and to examine variations and errors in the respective DEMs. The different DEMs and roughness maps from LiDAR collected at different densities are evaluated with a hydrodynamic flood model.

\subsection{Area investigated}

The catchment and floodplain of the Waikanae River on the West coast of southern North Island, New Zealand (Figure 1) was selected for this study for the following reasons:

- Existing LiDAR tiles with nominal 1 point $/ \mathrm{m}^{2}$ density were available.

- A hydrodynamic flood model was in development for this catchment.

- The range of topography, infrastructure and vegetation cover in the region represents a microcosm of conditions found across New Zealand.

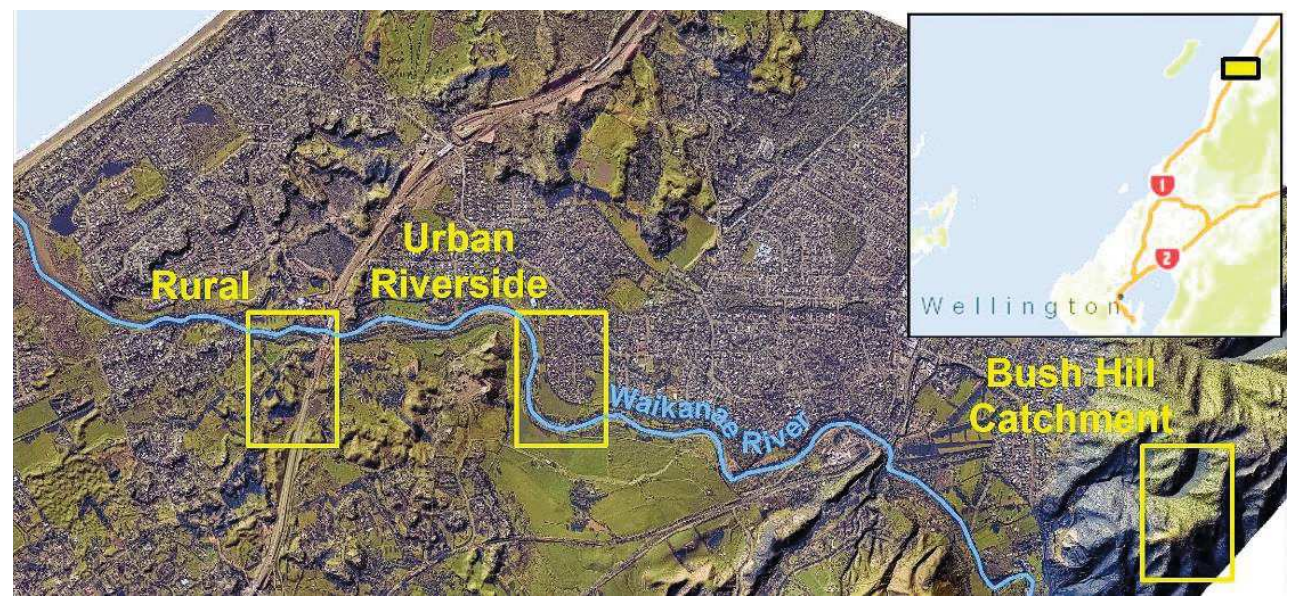

Fig. 1. Overview of part of Waikanae study site showing representative "Rural", "Urban Riverside" and "Bush Hill Catchment" LiDAR tile locations.

\subsection{LiDAR data}

LiDAR at nominal 1 point $/ \mathrm{m}^{2}$ density was supplied by Greater Wellington Regional Council. Collection at 4 and 8 points $/ \mathrm{m}^{2}$ was commissioned by Land Inventory New Zealand (LINZ) specifically for this study. The three data sets are called 'low', 'medium' and 'high' resolution in the following analyses.

The unrefined LiDAR point data was supplied in "tiles" measuring $480 \mathrm{~m}$ x $720 \mathrm{~m}$. Analysis of the data-sets revealed that the LiDAR point densities were significantly higher than the nominated densities. Table 1 shows actual point densities from a random sample of non-sequential LiDAR tiles.

Table 1. Nominal and actual LiDAR point densities.

\begin{tabular}{|r|c|c|c|}
\hline Quality & Low resolution & Medium resolution & High resolution \\
\hline Nominal density [points $/ \mathrm{m}^{2}$ ] & 1 & 4 & 8 \\
\hline Actual density range [points $/ \mathrm{m}^{2}$ ] & $0-16$ & $1-50$ & $6-251$ \\
\hline Most common number points $/ \mathrm{m}^{2}$ & 3 & 9 & Bimodal: $17 \& 29$ \\
\hline
\end{tabular}

The points for Table 1 density calculations refer to all LiDAR returns. This includes ground and non-ground hits. The average number of points $/ \mathrm{m}^{2}$ is much higher than the 
nominal specifications for the LiDAR survey and the point density within each data set is highly variable. The reason for high variability is, at least partially, due to the way the data is collected. To avoid gaps in the data, parallel LiDAR scans are flown to overlap each other and the overlapped areas contain around double the point density of areas with no overlap. The flight pattern during the collection of the nominal 8 point $/ \mathrm{m}^{2}$ data set contained additional flight lines oriented at 90 degrees which may explain the bimodal distribution of point densities (Table 1). This imprecise density should be taken into account if conclusions from this study are to be used for specification of LiDAR for modelling requirements.

To illustrate effects of the different LiDAR densities for different ground conditions, three sample LiDAR tiles were studied meticulously. These tile locations are shown on Fig. 1 and represent "Urban Riverside", "Rural", and "Bush Hill Catchment" situations.

\section{Analysis}

\subsection{Computational grid creation}

The supplied LiDAR tiles contained information indicating which of the hits were ground LiDAR points. As the LiDAR was sourced from different providers, there was no guarantee that the same ground-detection basis was applied to each of the data sets. Using "ground" attributed points could lead to confusion between differences caused by the grounddetection methodology and effects caused by point density. To avoid this possibility, it was decided to use a standard ground-selection algorithm across all unrefined data sets. The point cloud LiDAR was then processed into modelling grids as discussed below.

\subsection{1. $\mathrm{Z}$ and Zo extraction and gridding}

Individual point co-ordinates $(\mathrm{x}, \mathrm{y}, \mathrm{z})$ and reflected LiDAR intensity (i) of the point cloud data was analysed to extract ground elevation $(Z)$ and ground roughness $(\mathrm{Zo})$. This process used a search footprint. The ground elevation was determined to be the lowest $\mathrm{z}$ elevation within the footprint. The ground roughness was determined by an algorithm which analyses variation and absorption of returns within the local search footprint. The roughness algorithm was pre-trained for each LiDAR data sortie using reference surfaces such as roads, gravel areas, fields, scrub, etc. In order to correctly extract ground level and roughness there must be sufficient returns within a search footprint. The extraction process is computationally demanding because neighbouring LiDAR hits on the ground may be from different LiDAR flight path scans and widely separated in a data file.

Using the most common point densities from Table 1, Table 2 shows that search footprint radii of $1 \mathrm{~m}, 0.5 \mathrm{~m}$ and $0.375 \mathrm{~m}$ are suitable to achieve around 7-9 points per search footprint. This number of points is sufficient for roughness determination.

The result of the $\mathrm{Z}$ and Zo extraction process are $(\mathrm{x}, \mathrm{y}, \mathrm{Z})$ and $(\mathrm{x}, \mathrm{y}, \mathrm{Zo})$ data sets at the same density as the original data. These scattered data-sets are then gridded at the finest meaningful resolution, which is the footprint radius used for $\mathrm{Z}$ and $\mathrm{Zo}$ detection (Table 2).

\subsubsection{Upscaling}

The raw DEM and roughness grids are upscaled to a grid size appropriate for hydraulic modelling. The modelling grid size is a trade-off between a finer grid for resolving important features and a coarser grid for reducing model run-time. For flood inundation models of typical river systems, an underlying $3 \mathrm{~m}$ model grid is a realistic grid size choice. 
An upscaling ratio is used for each of the low, medium and high resolution footprint-sized grids to convert these raw grids to a 3 m modelling grid (see Table 2).

For upscaling of roughness, the average of underlying sub-grid values is used to set the upscaled grid value. For upscaling of ground elevations the average underlying value can be used but the averaging process smooths high and low points and does not necessarily preserve the level of natural barriers to flow such as crest elevations of stopbanks, or the invert level of overland flow paths or drains. One solution is to define break lines along critical features such as stopbanks or drains and enforce pre-defined breakline elevations on the upscaled grid DEM. This is usually a manual process which can miss unexpected crest lines such as roadways, railway embankments and vegetated swales.

Our methodology for preserving actual crest elevations during DEM upscaling was to apply an algorithm which sets the upscaled cell level to the lowest elevation at which water could spill across the underlying sub-grid cells. As an analogy, if the underlying cells are considered as a dam, the level of the upscaled cell is set to the spillway crest level of the dam (some models allow part blockage of a cell). Manual re-editing of the resulting DEM may be required where trees are located alongside narrow crest lines (Fig. 3), however, for an unbiased comparison of results across different LiDAR resolutions, no manual editing was undertaken in this study.

Table 2. Upscaling and gridding ratios.

\begin{tabular}{|c|c|c|c|}
\hline Quality & Low resolution & Medium resolution & High resolution \\
\hline Number of [points $/ \mathrm{m}^{2}$ ] & 3 & 9 & 17 \\
\hline Footprint radius used [m] & 1 & 0.5 & 0.375 \\
\hline Footprint area $\left[\mathrm{m}^{2}\right.$ ] & 3.14 & 0.785 & 0.441 \\
\hline Points/footprint area & 9.4 & 7.1 & 7.5 \\
\hline Raw grid size [m] & 1 & 0.5 & 0.375 \\
\hline Upscale factor & 3 & 6 & 8 \\
\hline Model grid size $[\mathrm{m}]$ & 3 & 3 & 3 \\
\hline
\end{tabular}

The result of the upscaling process is $3 \mathrm{~m}$ DEM grids (examples shown in Fig. 2) and 3 m roughness grids (Fig. 3) suitable for computational flood modelling applications. Fig. 2 shows that as the LiDAR resolution decreases, the true ground level is increasingly masked by vegetation (e.g. lower left trees on Fig. 2). Roughness extraction is less sensitive to resolution but some low roughness details are lost in the low res. LiDAR (Fig. 3, right image).

\subsection{Evaluation for a Rural area.}

The Rural area sample (Fig. 1) represents a rural landscape with rolling hills, isolated and rows of trees, wide open spaces as well as a small stream that is overhung by trees and bushes. Effects from construction of a road and bridge between the low res. Rural LiDAR and the medium and high res. Rural LiDAR were masked in comparisons of the data.

Difference maps and histograms (available separately and shown in oral presentation) were used to indicate discrepancies in the elevation and roughness $3 \mathrm{~m}$ grids for medium and low res. LiDAR, compared to high res. LiDAR. The medium LiDAR DEM was very close to the high res. grid with differences detected mainly along the edges of tree lines. The low res. DEM was also close to the high res. reference, except for vegetated river bed and shelter belt areas. For medium resolution, $84 \%$ of the elevations were within $300 \mathrm{~mm}$ of the high res. values and $62 \%$ were within $100 \mathrm{~mm}$. For low resolution, $75 \%$ of the elevations were within $300 \mathrm{~mm}$ of the high res. values and $51 \%$ were within $100 \mathrm{~mm}$. Roughness differences between the high and medium res. LiDAR were also mainly associated with edges of linear features. Some pasture areas in the medium res. LiDAR 
were smoother than indicated by the high res. LiDAR but this could be due to grazing in the interim. Differences between high and low res. LiDAR roughness shows that pasture areas have relatively similar roughness except where the underlying ground is steeper. On steeper slopes, larger footprint radii produce higher roughness than smaller footprints because of ground level variation due to the slope.

\subsection{Evaluation of Urban Riverside area.}

Part of the Urban Riverside area is shown in Figs. 2 and 3. For the medium res. LiDAR, discrepancies in elevation are not particularly significant and are primarily associated with vegetation. For the low res. LiDAR, discrepancies in elevation are pronounced in places. The stream bed is clearly lower, but this could be due to flow conditions or bed scouring in the interval between LiDAR surveys. The western floodplain is lower. There is underestimation of the levee crest level by 0.1-0.5 $\mathrm{m}$ (levee location is visible under the red + on Figure 2).

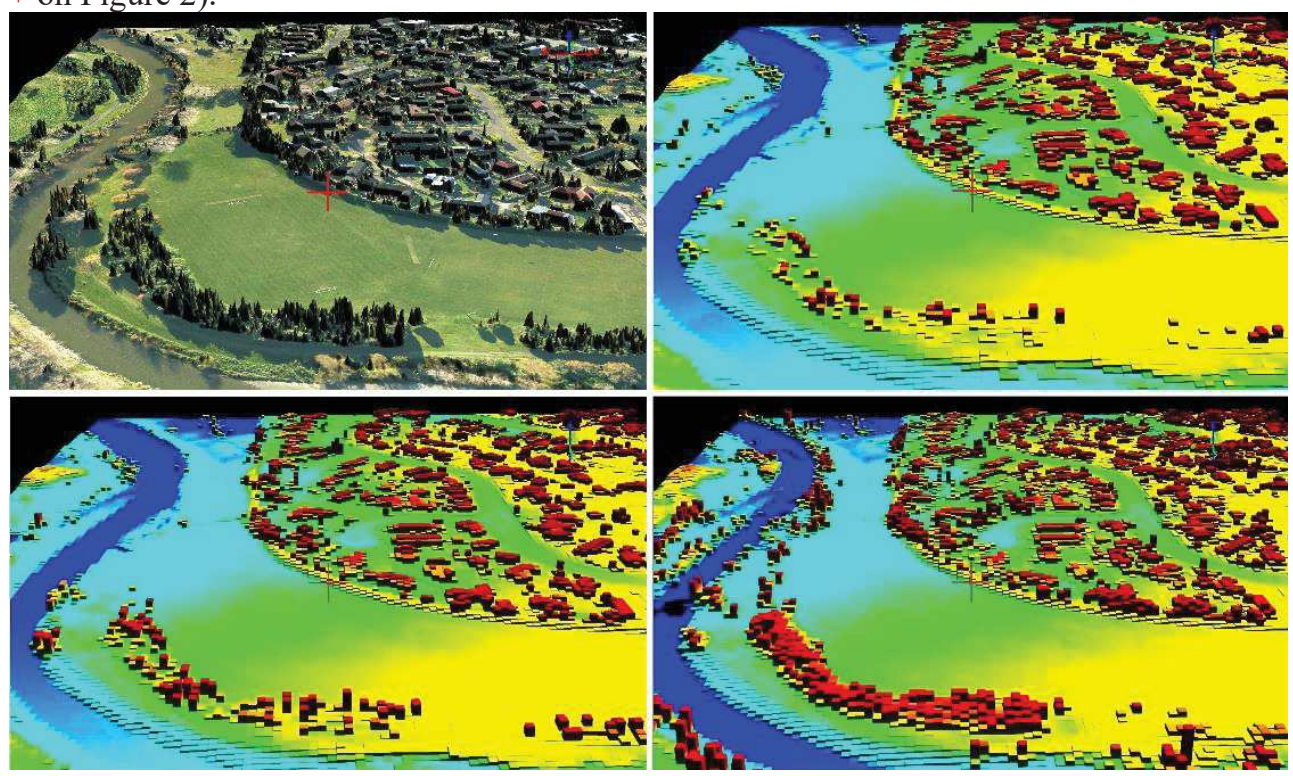

Fig. 2. Urban Riverside area showing air photo on raw LiDAR points (top left) and $3 \mathrm{~m}$ DEM grid derived from (clockwise) high, low and medium res. LiDAR. Grid elevations from low-high shown by blue $<$ green $<$ yellow $<$ red respectively. Black $=$ no data.
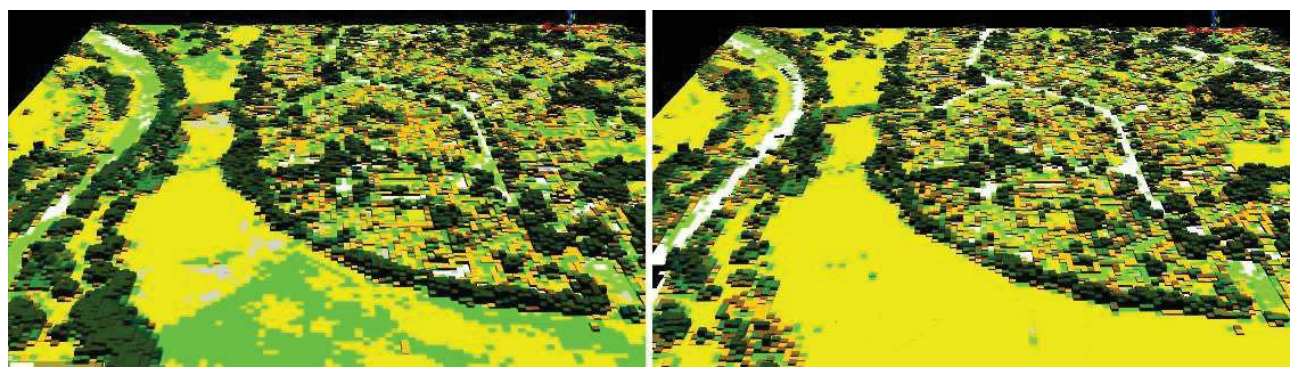

Fig. 3. Urban Riverside $3 \mathrm{~m}$ roughness grid derived from high resolution (left) and low resolution LiDAR (right). Roughness levels from low to high are shown by white $<$ grey $<$ yellow $<$ light-green $<$ orange $<$ brown $<$ dark-green colours. Black $=$ no data. The flood levee is covered by vegetation. 
For the medium res. LiDAR, discrepancies in roughness are evident and are primarily associated with vegetation and very smooth areas. On very smooth surfaces, such as roads, the roughness heights are as low as $1 \mathrm{~mm}$, so that a difference of $1 \mathrm{~mm}$ would indicate a relative error of $100 \%$. In addition, the velocity of floodwater is related to the logarithm of roughness height. Taking these factors into account, roughness from the 'medium' LiDAR appears to provide sufficient accuracy for flood modelling purposes. For the low res. LiDAR, discrepancies in roughness are pronounced. In the comparison with low res. LiDAR it should be borne in mind that this survey was undertaken several years earlier than the medium and high res. LiDAR. Discrepancies in vegetated areas are difficult to evaluate due to likely vegetation growth or decay in the period between the LiDAR surveys. However, the roughness discrepancies are significant, even in non-vegetated areas. There is a significant underestimation of roughness in the urban subdivision.

\subsection{Evaluation of Bush Hill area.}

The "Bush Hill" site has very different characteristics to the two other sites having steep slopes, dense forests and an intricate network of flow paths. The site also contains a broad grassed ridge. The different LiDAR density scans show wide, quasi random elevation discrepancies where there is dense bush cover. While the deviations can be of the order of a metre or more, considering the steep topography and the dense bush cover, these differences are considered tolerable. On the broad grassed ridges the discrepancies at the different resolutions are mainly within $\pm 0.1 \mathrm{~m}$ and all within $\pm 0.5 \mathrm{~m}$. The medium res. roughness is close to the high res. roughness except for a "block" in the upper part of the area where the medium res. roughness is lower. The low res. roughness is generally lower over the entire, densely vegetated parts of the Bush Hill tile. The exposed ridge has roughness within $5 \%$ of the high res. case. Examination of the block of lower roughness from the medium res. LiDAR reveals that the LiDAR scan density was much lower in this block, resulting in lower roughness as occurred with the low res. LiDAR. Considering that estimation of surface roughness below dense vegetation on steep terrain has inherently high uncertainty, the differences in roughness predicted with the different densities of LiDAR are considered tolerable. On the exposed ridges, the discrepancies at the different LiDAR resolutions are relatively low.

\subsection{Stream Network}

The continuity of streams was investigated for the Bush Hill site by calculating stream networks (continuous downhill flow paths) based on the $3 \mathrm{~m}$ DEMs derived from the high, medium and low density LiDAR surveys. The networks were compared with those from a $0.375 \mathrm{~m}$ DEM grid from the high res. LiDAR. In most areas the $3 \mathrm{~m}$ grid network is very close to the $0.375 \mathrm{~m}$ grid network.

The flow paths calculated from $3 \mathrm{~m}$ grids for the medium res. and low res. LiDAR DEMs can deviate from the reference by up to around $20 \mathrm{~m}$, however, for predicting runoff from the Bush Hill site it is not anticipated that the different channel networks would produce significantly different flows with the same rainfall input over the catchment.

\subsection{Continuity and crest heights}

Preserving the correct levels of flood control structures, such as weirs and levees, is important for inundation models. A relatively dense point spacing is required to accurately extract such features when they have narrow crests. 
On the Urban Riverside tile (Fig. 2) a levee along the right bank separates a developed housing area from the floodplain. Fig. 4 shows a 500m long profile along the levee crest extracted from the $3 \mathrm{~m}$ DEMs. The dark green line marks the high res. profile. From $130 \mathrm{~m}$ downstream to $500 \mathrm{~m}$ on the profiles, vegetation overhanging the bank has not been removed by the processing. This occurs when no LiDAR points in the footprint area penetrate through the vegetation canopy. With no vegetation the medium res. line fits the high res. line very well. The low res. profile is a lot noisier than the other two profiles.

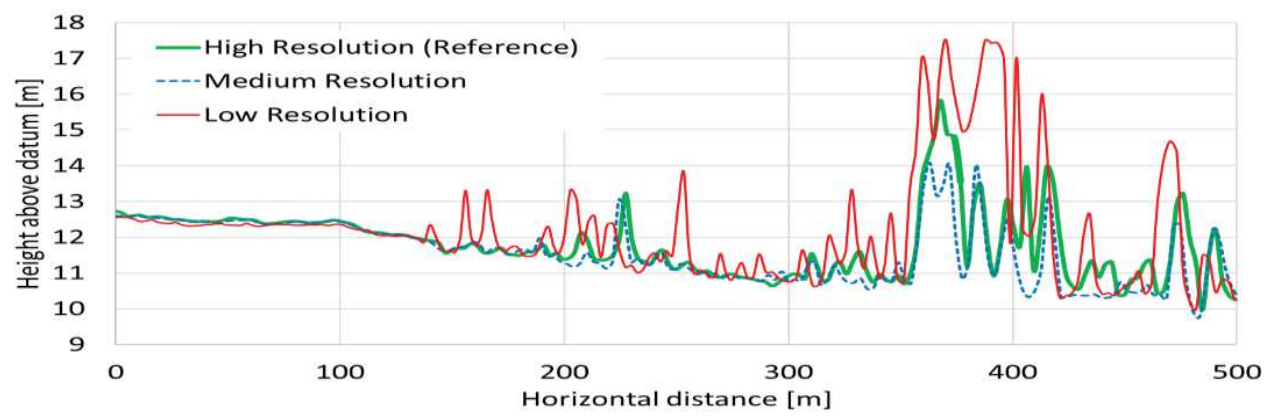

Fig. 4. Profiles along the crest of the levee which passes the red + on Fig. 2. The profiles indicate differences in the $3 \mathrm{~m}$ DEM grids made from the different quality LiDAR data sets.

While peaks from vegetation on this profile are not ideal, more concerning is a $0.2 \mathrm{~m}$ lowering where inundation models would indicate overtopping of the levees at a lower river level. Such troughs can be seen for the low res. data between $0 \mathrm{~m}-120 \mathrm{~m}$ distance along the profile. The medium res. graph also shows troughs in some places $(200 \mathrm{~m}-220 \mathrm{~m}, 420 \mathrm{~m}-$ $470 \mathrm{~m}$ ) which may represent the true crest level of the stop bank, since the high res. profile shows vegetation peaks in these areas.

Fig. 5 compares $3 \mathrm{~m}$ grid levels across a low swale and wire fence found west of the area shown in Fig. 2.

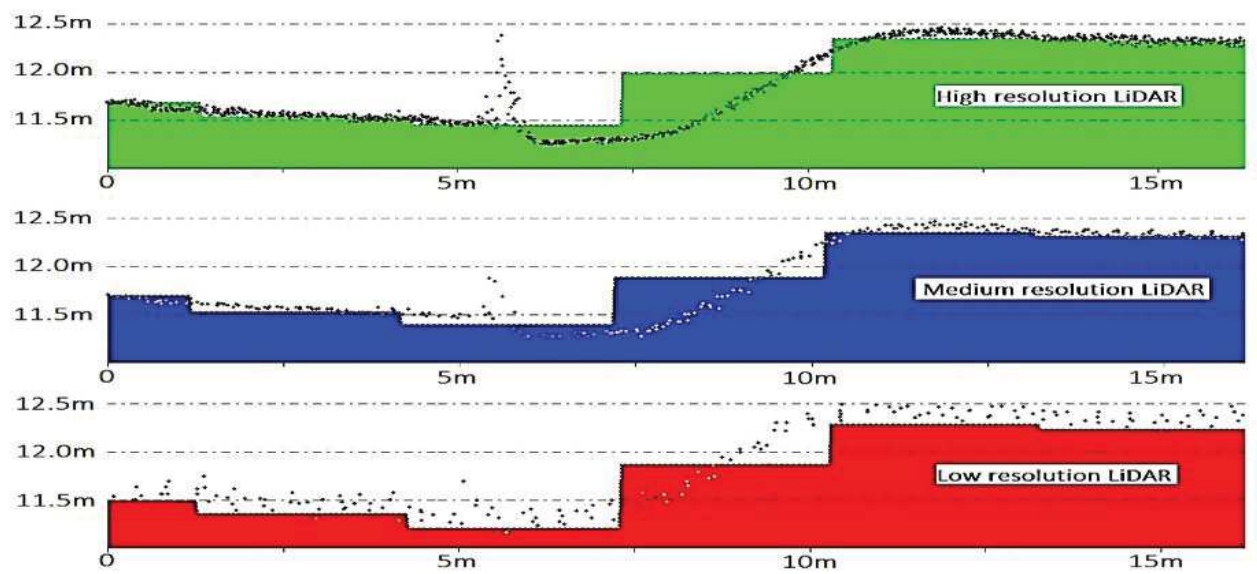

Fig. 5. Cross section comparing LiDAR hits and 3 metre grid surface level for high, medium and low resolution LiDAR. A wire fence exists at $5.5 \mathrm{~m}$ distance along the profile.

On Fig. 5, raw LiDAR hits are shown as points and the $3 \mathrm{~m}$ DEM grid by the coloured areas. The fence at distance $5.5 \mathrm{~m}$ along the cross-section is captured by the high res. LiDAR hits and partially by the medium res. LiDAR. The fence is not represented in any of the $3 \mathrm{~m}$ DEM grids. The low res. LiDAR produces a grid ground surface that is 0.1 to $0.2 \mathrm{~m}$ lower than the higher res. LiDAR grid. 


\subsection{Continuity and stream channel profile}

Continuity of streams and landscape features that can serve as conduits for flood water, is important for accurate inundation modelling. Obstructions to flow caused by virtual artefacts in DEMs can lead to serious errors in predicting flood hazard areas. Figure 6 shows profile lines along a section of a Rural area small stream that is partially overhung by trees and bushes for the various resolution DEMs.

The high res. LiDAR DEM (green line) contains some effects of overhanging trees which will block the flow of water along the channel if not corrected. The medium res. LiDAR elevations still follow the high res. profile fairly closely, slightly exaggerating the height of obstructions in the channel where dense vegetation is encountered. The low res. LiDAR underestimates the level of the stream bed where there is no vegetation $(0 \mathrm{~m}-150$ $\mathrm{m}$ ) and shows multiple locations where vegetation causes elevations higher than the other DEMs $(180 m-210 m, 260 m-310 m)$. The low res. LiDAR DEM produces erroneous high points which were not present in the other DEMs (i.e. $150 \mathrm{~m}-170 \mathrm{~m}, 330 \mathrm{~m}-350 \mathrm{~m}$ ).

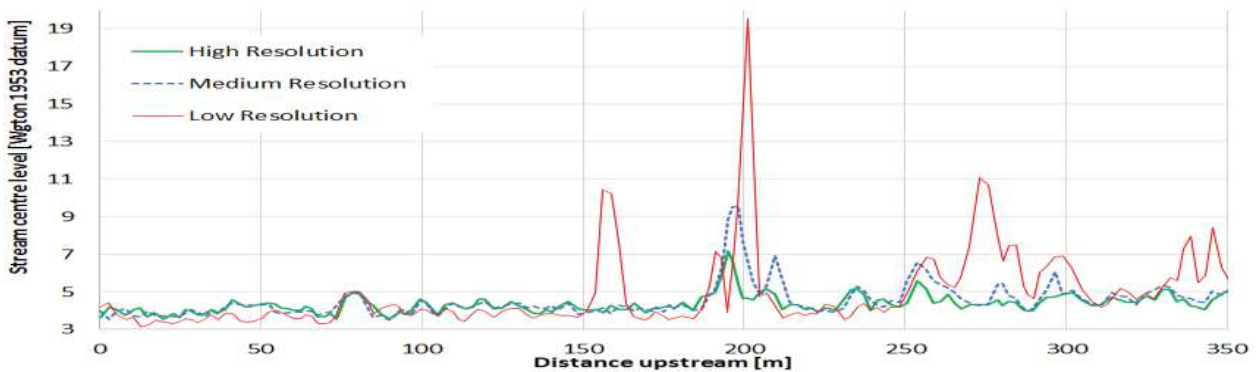

Fig. 6: Elevation profiles along the centre line of a small stream in the Rural area.

\subsection{Summary}

The medium res. LiDAR provided elevation and roughness grids sufficiently accurate for hydrodynamic flood mapping of urban and rural floodplains. Scrutiny may be required where narrow-crested control structures exist. The low res. LiDAR is considered adequate for hill catchments. Properties related to widely varying point density and multiple returns should be made clear if conclusions from this study are to be used to assist specification of LiDAR sortie requirements.

This research is funded by the New Zealand National Institute of Water \& Atmospheric Research (NIWA) and the NZ Natural Hazards Research Platform. Land Information New Zealand (LINZ) provided funding, advice and LiDAR for this study. Greater Wellington Regional council provided historic flood and background information.

\section{References}

1. G. Smart. Improving flood hazard prediction models, International Journal River Basin Management. Taylor \& Francis, DOI: 10.1080/15715124.2017.1411923 (2018)

2. A. Avanzi, E. Frank, M. Righetto, S. Fattorelli. LIDAR Data Resolution Versus Hydromorphological Models for flood risk assessment, The Role of Geomatics in Hydrogeological Risk, Int. Archives of Photogrammetry, Remote Sensing and Spatial Information Sciences, XL-5/W3, 119-124, (2013)

3. H. Rientjes. Effects of LIDAR DEM Resolution in flood modelling, A model sensitivity study for the city of Tegucigalpa. Laser scanning, ISPRS WG III/3, III/4, V/3 168-173, (2005) 